\title{
Expanding the phenotype of craniofrontonasal syndrome: two unrelated boys with EFNB1 mutations and congenital diaphragmatic hernia
}

\author{
Pradeep C Vasudevan*,1,4, Stephen RF Twigg ${ }^{2,4}$, John B Mulliken ${ }^{3}$, Jackie A Cook ${ }^{1}$, \\ Oliver WJ Quarrell ${ }^{1}$ and Andrew OM Wilkie ${ }^{2}$
}

${ }^{1}$ Department of Clinical Genetics, Sheffield Children's Hospital, Sheffield, South Yorkshire, UK; ${ }^{2}$ Weatherall Institute of
Molecular Medicine, John Radcliffe Hospital, Oxford, UK; ${ }^{3}$ Craniofacial Centre, Children's Hospital, Boston, MA, USA

Craniofrontonasal syndrome (CFNS, MIM 304110) is an X-linked craniofacial disorder that shows paradoxically greater severity in heterozygous females than in hemizygous males. Mutations have been identified in the EFNB1 gene that encodes a member of the ephrin-B family of transmembrane ligands for Eph receptor tyrosine kinases. Here, we describe two unrelated families, in both of which a mother and her son have proven mutations in EFNB1. The mothers have classical features of CFNS; although the sons have no major craniofacial features other than telecanthus, both had a congenital diaphragmatic hernia (CDH). Our cases represent the first in which CDH has been confirmed in males with mutations in EFNB1, highlighting an important role for signalling by ephrin-B1 in the development of the diaphragm.

European Journal of Human Genetics (2006) 14, 884-887. doi:10.1038/sj.ejhg.5201633; published online 26 April 2006

Keywords: craniofrontonasal syndrome; congenital diaphragmatic hernia; EFNB1

\section{Introduction}

In 1979, craniofrontonasal syndrome (CFNS, MIM 304110) was identified as a subgroup of frontonasal dysplasia by Cohen, ${ }^{1}$ who coined the term craniofrontonasal dysplasia. The condition is characterised in females by hypertelorism, coronal craniosynostosis, craniofacial asymmetry, frontal bossing, downslanting palpebral fissures, broad bifid nose, low posterior hairline with an anterior widow's peak, frizzy hair, occasional cleft lip and palate, longitudinally grooved fingernails and other digital anomalies. ${ }^{2-7}$ The fewer males described have mostly been more mildly affected with hypertelorism and, occasionally, cleft lip or palate. ${ }^{2-5,7}$ This greater severity in females than males has always

\footnotetext{
${ }^{*}$ Correspondence: Dr PC Vasudevan, Department of Clinical Genetics, Sheffield Childrens Hospital, Western Bank, Sheffield, SouthYorkshire, S10 2 TH, UK.

Tel: + 441142717025 or +44 114 2260570; Fax: + 44114 2737467;

E-mail: pradeepcv@yahoo.com

${ }^{4}$ These authors contributed equally to this work

Received 5 January 2006; revised 1 March 2006; accepted 1 March 2006; published online 26 April 2006
}

appeared surprising, given evidence from pedigree analysis that CFNS is an X-linked disorder. ${ }^{3,7}$

The X-linkage of CFNS was recently confirmed by the identification of mutations in the EFNB1 gene, which maps to Xq13.1, in both familial and sporadic cases. ${ }^{8-11}$ EFNB1 encodes ephrin-B1, a transmembrane ligand for Eph receptor tyrosine kinases that itself exhibits receptor-like effects. Significantly, mice with null mutations of ephrinB1 have malformations of the axial and appendicular skeleton that also show greater severity in heterozygous females than in either homozygous females or hemizygous males. ${ }^{12,13}$ Because of X-inactivation, heterozygous females are uniquely mosaic for cells that either do, or do not, make functional protein; this is proposed to lead to abnormal sorting of cell populations and disturbance of normal tissue boundary formation. The term cellular interference has been suggested for this process. $^{9}$ This ectopic signalling mechanism of pathogenesis makes it difficult to draw firm conclusions about the normal functions of ephrin-B1 from the phenotype of CFNS females. By contrast, the usually mild male phenotype 
associated with ephrin-B1 mutations in both humans and mice has been attributed to functional redundancy with other ephrins. Here we report a more severe phenotype, congenital diaphragmatic hernia (CDH), in two unrelated males hemizygous for EFNB1 mutations. This suggests a nonredundant role for ephrin-B1 in the development of the diaphragm.

\section{Case reports and mutation analysis Family 1}

A 4-year-old boy (case 1) born to nonconsanguineous Caucasian parents was found antenatally to have a left-sided CDH and sacrococcygeal teratoma. He was born by spontaneous normal vaginal delivery at 34 weeks' gestation. He had a primary repair of his diaphragmatic defect (Figure 1a) on day 2 with good result. His sacrococcygeal teratoma was excised on day 21 . On examination at 6 months of age, he had a prominent metopic suture, telecanthus (inner canthal distance of $28 \mathrm{~mm} ;+2$ s.d. above the mean), brachydactyly and pectus excavatum. Later he grew curly blonde hair (Figure 1b). There was no significant developmental delay. His head circumference and weight were between the 0.4 th and 2nd centile and height between the 2nd and 9th centile. Investigations revealed a normal male karyotype (46, XY). His cranial ultrasound scan and skull X-ray were normal. A clinical diagnosis of male CFNS was made on the basis of his mild dysmorphic features and characteristic clinical features in the mother (Figure 1c and d). Mutation analysis of EFNB1 using previously described methods ${ }^{8}$ demonstrated a frameshifting mutation (432delG, predicting L145WfsX14) in exon 3, which was heterozygous in the mother and hemizygous in her son (Figure 1e and f). While sacrococcygeal teratoma is not a recognised association of CFNS, we note that a similarly affected female CFNS patient with a confirmed EFNB1 mutation was described previously. ${ }^{8}$

\section{Family 2}

A male infant (case 2) of nonconsanguineous Caucasian parents was born at 37 weeks' gestation with left-sided $\mathrm{CDH}$, talipes equinovarus and swallowing difficulties. The $\mathrm{CDH}$ was repaired at age 3 days with a Gore-Tex patch, fundoplication and gastrostomy tube placement, with a good result. On examination at 2 years of age, he had telecanthus (inner canthal distance of $33 \mathrm{~mm}$; more than +2 s.d. above the mean) but no other dysmorphic features (Figure 2a). His mother (Figure 2b) and her two half sisters (Figure $2 \mathrm{c}$ and d), each of whom had different fathers, all had characteristic clinical features of CFNS. A clinical diagnosis of male CFNS was made in the proband on the basis of the hypertelorism and family history. Analysis of EFNB1 demonstrated a 3-nucleotide deletion in exon 2 (151_153delGTG, predict-
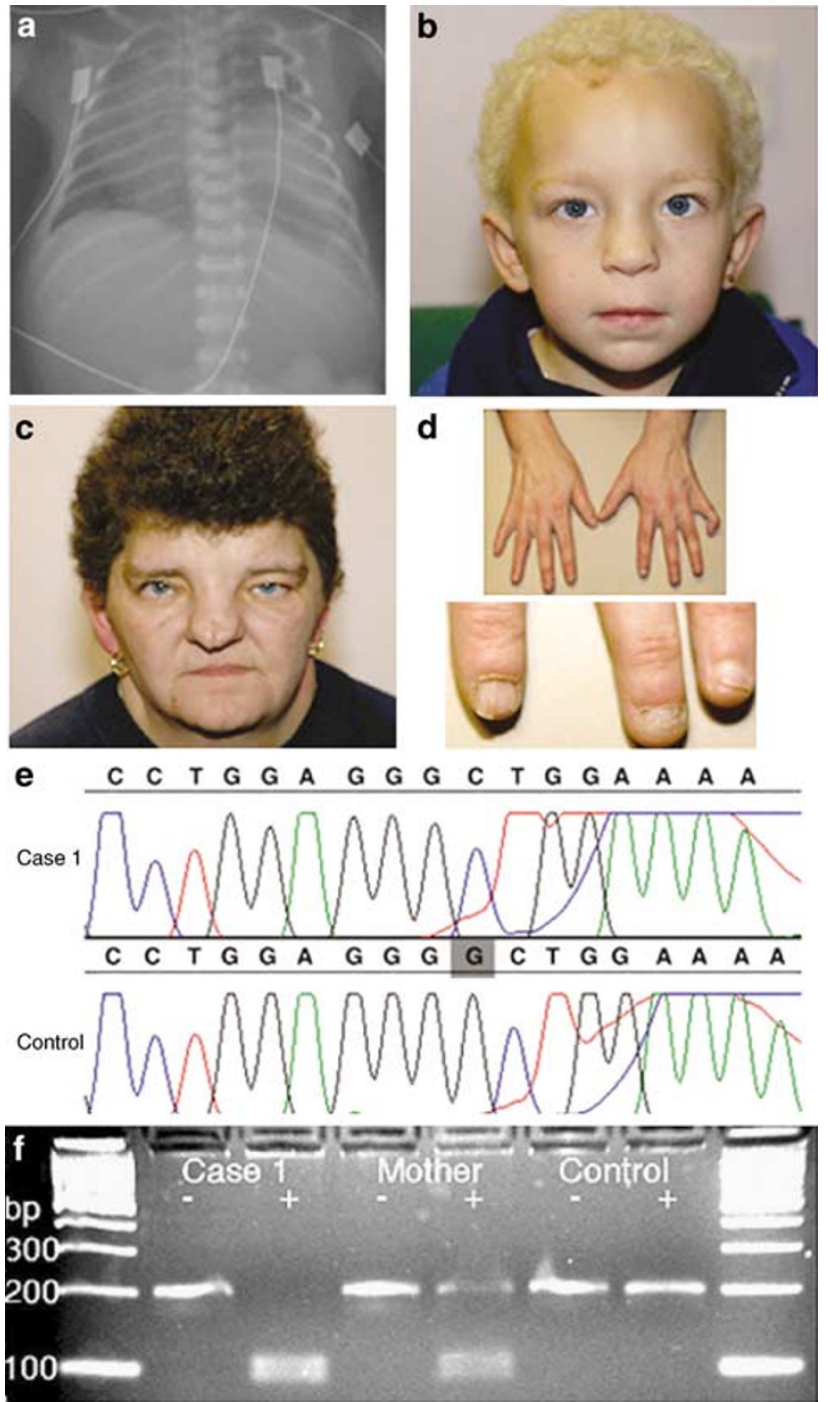

Figure 1 Clinical features and mutation analysis of case 1 and mother. (a) Plain radiograph of case 1 aged $3 \mathrm{~h}$. Note large left diaphragmatic hernia with stomach bubble in chest and gross mediastinal shift to right. (b) Case 1 aged 3 years. Note hypertelorism, prominent metopic ridge and curly hair. (c and d) Mother showing craniofacial asymmetry, telecanthus, broad bifid nose and digital anomalies with longitudinal ridging in nails. (e) Forward sequence of EFNB1 exon 3 from case 1 and control DNA. The nucleotide deleted in case 1 (432delG) is shaded in the control sequence. (f) Confirmation of 432delG mutation. EFNB1 exon 3 was amplified ${ }^{8}$ from case 1 , his mother and control DNA and incubated with Bgll, which specifically digests the 432delG allele (normal fragment $201 \mathrm{bp}$; mutant, 96 and $104 \mathrm{bp}$ ). Case 1 is hemizygous for the mutation, his mother is heterozygous and the control is negative. $(-)$ undigested, $(+)$ digested with Bgll.

ing V51del), present in heterozygous state in the mother and one of her sisters (a sample from the other sister was unavailable), and in hemizygous state in the son (Figure 2e and f). 

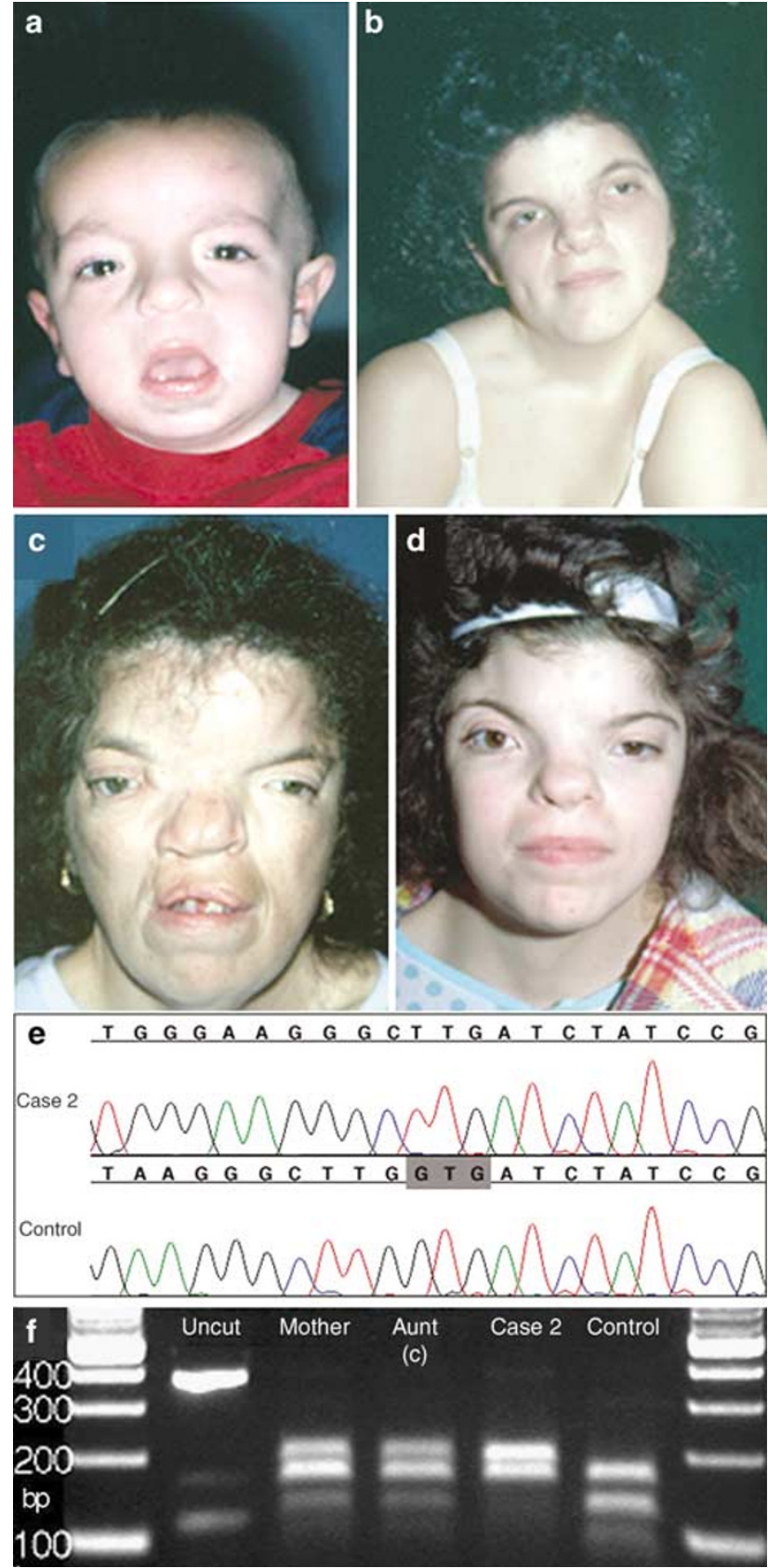

Figure 2 Clinical features and mutation analysis of case 2 and family. (a) Case 2 showing hypertelorism. (b) Mother of case 2 exhibiting craniofacial asymmetry and sloping shoulders. (c and d) Maternal aunts of case 2. (e) Reverse sequence of EFNB1 exon 2 from case 2 and control DNA. Three nucleotides (shown shaded in control) are deleted in case 2 (151_153delGTG). (f) Confirmation of 151_153delGTG mutation. EFNB 1 exon 2 was amplified ${ }^{8}$ and digested with $\mathrm{Hphl}$, a site for which is abolished by the mutation (normal allele 75,125 and $167 \mathrm{bp}$; mutant allele, 197 and $167 \mathrm{bp}$ ). The expected normal allele products are present in the control; the mother and aunt illustrated in panel c show the heterozygous pattern; case 2 is hemizygous for the mutation. PCR product not digested with $\mathrm{Hphl}$ (uncut) is $367 \mathrm{bp}$.

\section{Discussion}

The identification of two unrelated males hemizygous for EFNB1 mutations, both of whom have $\mathrm{CDH}$, provides new insight into the pathogenesis of this disorder. $\mathrm{CDH}$ occurs about once in 3000 newborns and is associated with a $30-60 \%$ mortality rate with significant morbidity among survivors. ${ }^{14} \mathrm{CDH}$ has been described in association with many chromosome abnormalities and in a number of syndromes; ${ }^{15-17}$ however, the only known genetic defect associated with nonsyndromic congenital diaphragmatic defect in humans is mutation in the FOG2 gene. ${ }^{18}$ Studies in rodents have shown three general causes of developmental error leading to $\mathrm{CDH}$. Failure of the normal closure of the pleuroperitoneal folds from embryonic day 12.5 in rats (equivalent to week 5 in humans) causes the common posterolateral defect seen in the cases described here. Alternatively, defects in the formation of the central tendon, or in migration of the muscle and nerve precursor cells from the cervical somites to the diaphragm, can also result in $\mathrm{CDH} .{ }^{19}$

$\mathrm{CDH}$ was previously described in four females with CFNS, ${ }^{8,20-22}$ but the developmental interpretation of these cases is complicated by the presence of X-inactivation (see Introduction). The only previous report of $\mathrm{CDH}$ in affected males was by Morris et $\mathrm{al}^{23}$ who described a family of six individuals with apparent CFNS including two affected brothers with $\mathrm{CDH}$. However, several authors have disputed this diagnosis as only one family member had craniosynostosis and the affected males had additional manifestations not described in CFNS, including short stature, delayed bone age, shawl scrotum and hypospadias; an alternative diagnosis of brachycephalofrontal dysplasia (Teebi syndrome; MIM 145420) has been proposed. ${ }^{5,7,22}$ To our knowledge, no molecular analysis has been undertaken in this family, so the issue remains unresolved. The two male $\mathrm{CDH}$ cases presented here represent the first in which the diagnosis has been confirmed by the finding of pathogenic mutations in EFNB1. Both mutations are likely to lead to the loss of function. In family 1 , the frameshift mutation truncates the C-terminal portion of the extracellular domain and, in addition, may lead to nonsensemediated decay of the mutant transcript; in family 2 , the deleted valine is within a $\beta$-sheet ${ }^{24}$ and therefore normal folding of the protein is likely to be disrupted. The spectrum of EFNB1 mutations observed in males without $\mathrm{CDH}^{9-11}$ is qualitatively similar, arguing against a distinct pathogenic action of the mutations observed here: presumably, the occurrence of $\mathrm{CDH}$ in our cases was caused by additional, unknown modifier effects.

In conclusion, our data indicate that the male CFNS phenotype is not always mild and that loss of ephrin-B1 function may interfere with normal diaphragmatic development. These observations need to be incorporated into genetic counselling for families with CFNS. Previous studies of ephrin-B1 expression in mouse ${ }^{25}$ and chick ${ }^{26}$ 
do not indicate which aspects of diaphragmatic development might be affected, and diaphragmatic defects were not reported in either of the published murine Efnb1 mutants. ${ }^{14,15}$ Our observations provide impetus to further experimental studies of ephrin-B1 signalling in the development of the diaphragm.

\section{Acknowledgements}

We are very grateful to the families for their participation in this work. The mutation analysis described in this work was funded by the Wellcome Trust (AOMW).

\section{References}

1 Cohen Jr MM: Craniofrontonasal dysplasia. Birth Defects Orig Art Ser 1979; 15: 85-89.

2 Slover R, Sujansky E: Frontonasal dysplasia with coronal craniosynostosis in three sibs. Birth Defects Orig Art Ser 1979; 15: 75-83.

3 Grutzner E, Gorlin RJ: Craniofrontonasal dysplasia: phenotypic expression in females and males and genetic considerations. Oral Surg Oral Med Oral Pathol 1988; 65: 436-444.

4 Kere J, Ritvanen A, Marttinen E, Kaitila I: Craniofrontonasal dysostosis: variable expression in a three-generation family. Clin Genet 1990; 38: 441-446.

5 Saavedra D, Richieri-Costa A, Guion-Almeida ML, Cohen Jr MM: Craniofrontonasal syndrome: study of 41 patients. Am J Med Genet 1996; 61: 147-151.

6 Orr DJA, Slaney S, Ashworth GJ, Poole MD: Craniofrontonasal dysplasia. Br J Plast Surg 1997; 50: 153-161.

7 Cohen Jr MM: Syndromes with craniosynostosis-craniofrontonasal syndrome; in Gorlin RJ, Cohen Jr MM, Hennekam RCM (ed): Syndromes of the Head and Neck. Oxford: Oxford University Press, 2001, pp 981-983.

8 Twigg SRF, Kan R, Babbs C et al: Mutations of ephrin-B1 (EFNB1), a marker of tissue boundary formation, cause craniofrontonasal syndrome. Proc Natl Acad Sci USA 2004; 101: 8652-8657.

9 Wieland I, Jakubiczka S, Muschke P et al: Mutations of the ephrinB1 gene cause craniofrontonasal syndrome. Am J Hum Genet 2004; 74: $1209-1215$.

10 Wieland I, Reardon W, Jakubiczka S et al: Twenty-six novel EFNB1 mutations in familial and sporadic craniofrontonasal syndrome (CFNS). Hum Mutat 2005; 26: 113-118.
11 Twigg SRF, Matsumoto K, Kidd AMJ et al: The origin of EFNB1 mutations in craniofrontonasal syndrome: frequent somatic mosaicism and explanation of the paucity of carrier males. Am J Hum Genet 2006 (in press).

12 Compagni A, Logan M, Klein R, Adams R: Control of skeletal patterning by ephrinB1-EphB interactions. Dev Cell 2003; 5: 217-230.

13 Davy A, Aubin J, Soriano P: Ephrin-B1 forward and reverse signalling are required during mouse development. Genes Dev 2004; 18: 572-583.

14 Harrison MR, Adzick NS, Estes JM, Howell LJ: A prospective study of the outcome for fetuses with diaphragmatic hernia. JAMA 1994; 271: 382-384.

15 Witters I, Legius E, Moerman P et al: Associated malformations and chromosomal anomalies in 42 cases of prenatally diagnosed diaphragmatic hernia. Am J Med Genet 2001; 103: 278-282.

16 Lurie IW: Where to look for the genes related to diaphragmatic hernia? Genet Couns 2003; 14: 75-93.

17 Slavotinek AM: The genetics of congenital diaphragmatic hernia. Semin Perinatol 2005; 29: 77-85.

18 Ackerman KG, Herron BJ, Vargas SO et al: Fog2 is required for normal diaphragm and lung development in mice and humans. PLoS Genet 2005; 1: 58-65.

19 Babiuk RP, Zhang W, Clugston R et al: Embryological origins and development of the rat diaphragm. J Comp Neurol 2003; 455: $477-487$.

20 Hurst J, Baraitser M: Craniofrontonasal dysplasia. J Med Genet 1988; 25: $133-138$

21 Brooks AS, van Dooren M, Hoogeboom J et al: Congenital diaphragmatic hernia in a female patient with craniofrontonasal syndrome. Clin Dysmorphol 2002; 11: 151-153.

22 McGaughran J, Rees M, Battin M: Craniofrontonasal syndrome and diaphragmatic hernia. Am J Med Genet 2002; 110: 391-392.

23 Morris CA, Palumbos JC, Carey JC: Delineation of the male phenotype in craniofrontonasal syndrome. Am J Med Genet 1987; 27: $623-631$

24 Nikolov DB, Li C, Barton WA, Himanen JP: Crystal structure of the ephrin-B1 ectodomain: implications for receptor recognition and signaling. Biochemistry 2005; 44: 10947-10953.

25 Flenniken AM, Gale NW, Yancopoulos GD, Wilkinson DG: Distinct and overlapping expression patterns of ligands for Ephrelated receptor tyrosine kinases during mouse embryogenesis. Dev Biol 1996; 179: 382-401.

26 Baker RK, Antin PB: Ephs and ephrins during early stages of chick embryogenesis. Dev Dyn 2003; 228: 128-142. 